\title{
Dairy consumption and risk of falls in 2 European cohorts of older adults
}

Short running head: Dairy consumption and risk of falls in older adults

Marcos D. Machado-Fragua, ${ }^{1}$ Ellen A. Struijk, ${ }^{1}$ Francisco Félix Caballero, ${ }^{1}$ Rosario Ortolá, ${ }^{1}$ Alberto Lana, ${ }^{2}$ José R. Banegas, ${ }^{1}$ Fernando Rodríguez-Artalejo, ${ }^{1,3}$ Esther Lopez-Garcia ${ }^{1,3}$

1. Department of Preventive Medicine and Public Health. School of Medicine. Universidad Autónoma de Madrid; IdiPaz (Instituto de Investigación Sanitaria Hospital Universitario La Paz); and CIBERESP (CIBER of Epidemiology and Public Health), Madrid, Spain.

2. Department of Medicine. School of Medicine and Health Sciences. Universidad de Oviedo / ISPA, Spain.

3. IMDEA-Food Institute. CEI UAM+CSIC, Madrid. Spain.

Main text word count: 3729

Number of data elements: 4

Number of supplemental elements: 6

Address for correspondence:

Esther Lopez-Garcia, PhD.

Department of Preventive Medicine and Public Health

School of Medicine

Universidad Autónoma de Madrid

C/ Arzobispo Morcillo, s/n

28029 Madrid, Spain

E-mail: esther.lopez@uam.es

Phone number: +34914972738 


\section{ABSTRACT}

2 Background \& aims: Some previous evidence have linked dairy products with greater

3 muscle mass, bone mineral density and lower risk of osteoporosis. However, other

4 authors have found a detrimental effect of milk on the risk of hip fracture. The aim of

5 this study was to assess the prospective association between dairy consumption and risk

6 of falls in older adults.

7 Methods: We used data from 2 cohorts of community-dwellers aged $\geq 60 \mathrm{y}$ : the Seniors-

8 ENRICA cohort with 2,981 individuals, and the UK Biobank cohort with 8,927

9 participants. In the Seniors-ENRICA, dairy consumption was assessed with a validated

10 diet history in 2008-10, and falls were ascertained up to 2015. In the UK Biobank study,

11 dairy consumption was obtained with 3-5 multiple-pass 24-h food records in 2006-10,

12 and falls were assessed up to 2016.

13 Results: A total of 801 individuals in the Seniors-ENRICA and 201 in the UK Biobank

14 experienced $\geq 1$ fall. After adjustment for potential confounders, dairy products were not

15 associated with risk of falls in the Seniors-ENRICA [hazard ratio (95\% confidence

16 interval) per 1-serving increment in total dairy consumption: 1.02 (0.93-1.11), milk:

170.93 (0.85-1.01), yogurt: $1.05(0.96-1.15)$, and cheese: $0.96(0.88-1.05)]$. Corresponding

18 figures in the UK Biobank were: total dairy: 1.19 (1.00-1.41), milk: 1.53 (1.13-2.08),

19 yogurt: $1.10(0.90-1.31)$, and cheese: $1.02(0.87-1.22)$.

20 Conclusions: These results suggest a null association between habitual dairy consumption and the risk of falling in older adults. Whether milk consumption may increase the risk of falls, as observed in the UK Biobank cohort, merits further study.

23 Keywords: older adults, epidemiology, milk, yogurt, cheese. 
Falls in older persons are a major public health problem, as they increase the risk of many adverse health outcomes and death (1). However, studies that evaluate the association between diet and risk of falling are scarce, so only a few of them have examined the role of nutrients, such as calcium (2), vitamin D $(2,3)$ and proteins (4), diet patterns (5) and individual foods $(6,7)$.

Dairy products are an important source of energy, proteins, vitamins and minerals, including calcium, potassium and vitamin $\mathrm{D}$, which are important nutrients for bone and muscle health (8). Previous studies have linked dairy products with a lower risk of frailty (9), cardiovascular disease $(10,11)$ and type 2 diabetes $(12,13)$, which are also predictors of falls in older people. Furthermore, in a cross-sectional study, a higher dairy consumption was associated with lower prevalence of falls, greater lean body mass and better physical performance in women (14). Likewise, dairy products have been associated with greater muscle mass $(14,15)$ and greater bone mineral density (16), as well as with lower risk of osteoporosis and functional disability (17-20). These results might suggest that dairy intake could prevent falls through the improvement of the musculoskeletal system. On the other hand, some authors have hypothesized a detrimental effect of milk on the risk of hip fracture $(19,21)$ because of its content of Dgalactose; however, this hypothesis has not been confirmed in a recent study (22). Lastly, since each type of dairy product provides different amounts of nutrients and bioactive compounds, they might also have different effects on health (10).

The aim of this study was to evaluate the prospective association between dairy products consumption, specifically, total dairy, milk, yogurt and cheese, and risk of 
48 from Spain and the United Kingdom (UK), to better understand the underlying role of 49 population characteristics and to improve the external validity of the results. 


\section{Study design and participants}

\section{The Seniors-ENRICA study}

The ENRICA study (Study on Nutrition and Cardiovascular Risk in Spain) was established in 2008-2010 with 12,948 individuals representative of the noninstitutionalized population of Spain aged $\geq 18 \mathrm{y}$ (23). Of them, 3,289 participants aged $\geq 60 y$ comprised the Seniors-ENRICA cohort. Information about socio-demographic characteristics, lifestyle, health status and morbidity were collected at baseline through a telephone interview. Moreover, two home visits were performed to assess food consumption, conduct a physical examination and obtain blood and urine samples. Two waves of data collection were subsequently performed in 2012 and 2015 to update the information of the cohort. The Clinical Research Ethics Committee of 'La Paz' University Hospital in Madrid approved the study protocol, and the study participants gave their informed written consent.

\section{The UK Biobank study}

The UK Biobank cohort has recruited more than half a million individuals between 4069 years $(81,725$ were $\geq 60 y)$ in the UK during the period 2006-2010 (24). At baseline, participants completed a touchscreen questionnaire, conducted a face-to-face interview, underwent a physical exam, and provided biological samples. Two waves of data collection (in 2012-2013 and 2014-2016) have been performed in a subsample of participants to update the information of the cohort. The UK Biobank study was conducted under generic ethical approval from the NHS National Research Ethics Service (ref 11/NW/0382, 17 June 2011).

\section{Study variables}


In the Seniors-ENRICA, dietary information was collected through a validated electronic diet history developed from the one used in the European Prospective Investigation into Cancer and Nutrition cohort study in Spain (25). This method of diet assessment included 880 different foods and allows for taking different portion sizes and cooking methods into account, as well as weekly and seasonal variations in food consumption. The dairy products recorded were: whole milk, part-skim and skim milk, whole- and low-fat yogurt, and cheese. We transformed the quantity consumed ( $\mathrm{ml}$ or $\mathrm{g}$ per day) into serving/day, by dividing the total amount of each food by the standard serving size (200 ml of milk, $125 \mathrm{~g}$ of yogurt and $40 \mathrm{~g}$ of cheese) $(9,22)$. In the validation study we found a moderate to good correlation between consumption of dairy products estimated from the diet history and the mean of seven 24-hour recalls during one year $(\mathrm{r}=0.68)$. Total energy intake and calcium, protein, and saturated fat were estimated using standard food composition tables (25) and were adjusted for energy intake using the residual method (26). Adherence to the Mediterranean diet was assessed with the Mediterranean Diet Adherence Screener (MEDAS) (27). This score includes 14 items on food consumption and food intake habits characteristic of the traditional Mediterranean diet in Spain. The MEDAS score ranges from 0 to 14, with a higher score indicating greater adherence (Supplemental table 1).

In the UK Biobank, food consumption was assessed through five web-based 24-h recalls (Oxford WebQ) (28), which included more than 200 foods frequently consumed in the UK and allowed for considering seasonal variations. For the present analyses, we selected the participants who completed at least three 24-h recalls. Dairy products recorded were: whole milk, part-skim and skim milk, powdered milk, full- and low-fat yogurt, and cheese. The reported consumptions for each type of dairy were: none, $0.5,1$, 
$2,3,4,5$ and $\geq 6$ servings/day. The mean of consumption in servings/day was calculated among the available 24-h recalls for each participant. Nutrient intakes were estimated with standard foods composition tables in the UK (29) and adjusted for energy by the residual method. Finally, adherence to the Mediterranean diet as per the MEDAS score was also assessed in this population as an indicator of diet quality. Two items in the score were excluded for not having this information in the cohort: the item on the amount of olive oil consumed, and the item related to "sofrito" (a type of cooking that uses a sauce made with fried tomato and vegetables). Thus, in the UK Biobank the MEDAS score ranged from 0 to 12 (Supplemental table 1).

\section{Falls}

In the Seniors-ENRICA study, self-reported incident falls were assessed by asking the participants at the follow-up waves (2012 and 2015): "How many times have you fallen down since the last interview?" We categorized the answers into: having no falls, and $\geq 1$ fall. In addition, participants reported if because of the fall, they had suffered a fracture. In the UK Biobank study, information about self-reported falls was obtained from the touchscreen questionnaire at baseline and the subsequent two follow-up waves by asking the participants: "In the last year have you had any falls?" The possible answers were "no falls", "only one fall", and "more than one fall". This variable was also categorized into: having no falls, and $\geq 1$ fall.

\section{Mortality}

In the Seniors-ENRICA study, we performed a computerized search of the National Death Index to evaluate all-cause mortality (30). This information was available for 99.9\% of the cohort. In the UK Biobank study, death certificates from the National 
122 Health Service (NHS) Information Centre (England and Wales) and the NHS Central

123 Register Scotland (Scotland) provided information about vital status (31).

124

125

126

127

128

\section{Other variables}

In the Seniors-ENRICA, data on age, sex, educational level $(\leq$ primary, secondary, university), smoking status (never, former, current smoker) and alcohol intake (abstainer: $<0.1 \mathrm{~g} / \mathrm{d}$, moderate drinker: $0.1-39 \mathrm{~g} / \mathrm{d}$ in men and 0.1-23 g/d in women, and heavy drinker: $\geq 40 \mathrm{~g} / \mathrm{d}$ in men and $\geq 24 \mathrm{~g} / \mathrm{d}$ in women) (32) were collected at baseline. Weight and height were measured in each participant under standardized conditions. Body mass index (BMI) was calculated as weight $(\mathrm{kg})$ divided by the squared height (m). Physical activity during leisure time (metabolic equivalent tasks-h/week) was evaluated with the EPIC-cohort questionnaire, validated in Spain (33). Self-reported sleep duration (h/d) was also recorded. Blood pressure (BP) was measured with a validated sphygmomanometer using standardized procedures, and hypertension was defined as systolic $\mathrm{BP} \geq 140 \mathrm{~mm} \mathrm{Hg}$, diastolic $\mathrm{BP} \geq 90 \mathrm{~mm} \mathrm{Hg}$, or being under hypertensive drug treatment. Twelve-hour-fasting serum glucose was measured, and type 2 diabetes was defined as glucose $\geq 126 \mathrm{mg} / \mathrm{dl}$ or being on oral antidiabetic drugs or insulin. Participants were considered frail when they met three or more of the following five self-reported criteria proposed by Morley et al. (34): fatigue, reduced resistance, reduced aerobic capacity, having several illnesses and a significant weight loss during the previous. Participants also reported if they had been diagnosed with osteo-muscular disease (osteo-arthritis or arthritis) by a physician. Finally, use of sleeping pills was assessed.

In 2013, several other measurements were performed to assess sarcopenia in this population. Percentage of body fat (\%BF) was measured by bioelectrical impedanciometry and skeletal muscle mass (SMM) with the equation developed by 
147 Janssen (35). Skeletal muscle mass index (SMI) was obtained by dividing SMM by

148 height squared. Muscle strength was approached through grip strength, considering the

149 highest value of two consecutive measures on the dominant hand using a Jamar

150 dynamometer. Physical performance was measured using the Short Physical

151 Performance Battery (SPPB), following the protocols of the National Institute on Aging 152 (36).

153 In the UK Biobank study, most of the variables were collected and categorized as in

154 Seniors-ENRICA study. However, physical activity was ascertained with questions

155 from the short International Physical Activity Questionnaire (IPAQ) (37) and the

156 presence of diabetes, hypertension or osteo-muscular disease was self-reported by the

157 participants.

\section{Statistical analyses}

159 In the Seniors-ENRICA, we excluded 308 participants: 4 with missing data on dairy consumption, 13 with implausible values of energy intake (outside the range of 800$5,000 \mathrm{kcal} / \mathrm{d}$ for men and 500-4,000 kcal/d for women), and 291 who were frail or lacked data on this variable at baseline (because frailty is a strong predictor of falls) (38) In the UK Biobank, among those $\geq 60 \mathrm{y}$, we excluded 72,798 participants: 69,476 with $<3$ web-based 24-h diet recalls, 137 with missing data on dairy, 939 with implausible high or low energy intake, and 2,246 who reported falls or lacked data on falls at baseline. This resulted in an analytical sample of 2,981 individuals in the SeniorsENRICA and 8,927 in the UK Biobank study (Supplemental figure 1).

We categorized dairy products consumption into tertiles of servings/day, except for milk in the UK Biobank study, which was categorized in two groups (yes vs no 
171 reference for the analyses. Person-years of follow-up were calculated from the date of

172 the baseline questionnaire until the date of the outcome (falling), death, loss to follow-

173 up, or the end of the study, whichever came first. To assess the association between

174 categories of dairy consumption and the incidence of falls, we used Cox regression

175 models to estimate hazard ratios (HR) with 95\% confidence intervals (CI) for each

176 category of dairy consumption. We built several models: the first one adjusted for age

177 and sex; the second one additionally adjusted for other potential confounders, including

178 education, smoking, alcohol intake, BMI, physical activity, sleep duration, energy

179 intake, MEDAS score, hypertension, diabetes and use of sleeping pills; and a third

180 model further adjusted for osteo-muscular disease, to explore its role in the studied

181 relationship. Moreover, when we assessed the specific association for milk, yogurt, and

182 cheese, we built a fourth model additionally adjusted for the other types of dairy

183 products. To investigate the linear dose-response relation, we modeled the categories of

184 dairy products consumption as continuous variables. Likewise, we calculated the risk of

185 falls associated with a 1-serving/d increment for the different types of dairy. We also

186 performed some sensitivity analyses by excluding participants with cardiovascular

187 disease, hip fracture and those who were heavy alcohol drinkers at baseline since they

188 had higher risk of falling than other population subgroups. This analysis was not

189 possible to be done for milk consumption and risk of falling in UK Biobank due to the

190 low number of cases among milk consumers. We also adjusted our main analyses by

191 markers of sarcopenia status (percentage of lean mass, handgrip strength and gait speed)

192 to explore the implication of sarcopenia in the studied association in the Seniors-

193 ENRICA cohort, which had these measurements done. Moreover, we analyzed the

194 relationship between the different dairy products and the risk of falls with fracture in the

195 Seniors-ENRICA study. We did not use data on falls with fractures in the UK Biobank 
cohort due to the very small number found $(n=19)$. Finally, in order to account for changes in dairy consumption during follow-up, we estimated the cumulative consumption by using dietary information available in Seniors-ENRICA at baseline and in the first follow-up wave, 2 years later (in the UK Biobank study, repeated diet measurement was not available).

201

The main analyses were stratified by sex, hypertension, type 2 diabetes, osteo-muscular disease, sleep duration, protein intake and physical activity; also, likelihood-ratio tests comparing models with and without interactions terms were used to assess if results varied across strata. These analyses were performed only in the Seniors-ENRICA since in the UK Biobank the number of falls was too low.

206 Finally, to test the non-linear trends of risk of falls according to total dairy products 207 consumption, we used restricted cubic-splines with three knots. We conducted the 208 analyses separately in each cohort using Stata (version 15.0; Stata Corp., College 209 Station). 


\section{RESULTS}

211 Characteristics of the participants in each cohort according to tertiles of total dairy

212 consumption are presented in Table 1. In the Seniors-ENRICA, participants in the

213 highest vs. lowest tertile were less frequently men and less often current smokers or

214 heavy drinkers. Also, they had a higher BMI, a higher intake of energy, calcium,

215 protein, and saturated fat, and a lower adherence to the Mediterranean diet. The

216 distribution of dairy consumption was similar in the UK Biobank, with the exception

217 that those with higher consumption also had higher education and adherence to the

218 Mediterranean diet.

219 The intake of both total dairy and each type of dairy products was higher in Spain than

220 in the UK, except for low fat yogurt (Table 2). Average total dairy intake was 2.28

221 (standard deviation: 1.36) servings/d among participants in the Seniors-ENRICA, and

$2220.92(0.64)$ servings/d in those in the UK Biobank. Milk accounted for a large part of

223 this consumption in Spain (45\%) but not in UK (19\%). In addition, part-skim milk was

224 the most consumed type of milk in both cohorts (40\% and 67\%, respectively).

225 In the Seniors-ENRICA study, over 7.2 years of follow-up (median follow-up, 5.4 y),

226801 individuals reported $\geq 1$ falls (Table 3). No association was found between the

227 consumption of total dairy or any type of dairy products and the risk of falls, neither in

228 the crude- nor in the fully-adjusted analyses [fully-adjusted hazard ratio (95\%

229 confidence interval) per 1-serving/d increment in total dairy consumption: 1.02 (0.93-

230 1.11), milk: 0.93 (0.85-1.01), yogurt: 1.05 (0.96-1.15), and cheese: $0.96(0.88-1.05)]$.

231 Neither we found any difference in this association by categories of sex, morbidity,

232 sleep duration, protein intake and physical activity level ( $\mathrm{p}$ for interaction $>0.05$ in all

233 cases) (Supplemental table 2). Moreover, we did not find any association between 
dairy products and the risk of falls with fracture (Supplemental table 3). Also, when cumulative dairy consumption was used as exposure, we still observed a null association with falls risk (Supplemental table 4). Similar results were also obtained when we excluded participants with cardiovascular disease, hip fracture and those who were heavy alcohol drinkers at baseline, or when we adjusted our analyses by markers of sarcopenia status (Supplemental tables 5 y 6).

Over 10.2 years of follow-up in the UK Biobank study (median follow-up, 3.2 y), 201 people reported $\geq 1$ falls (Table 4). Participants in the highest tertile of total dairy consumption showed a higher risk of falls in the crude model [HR: 1.51 (95\% CI: 1.07, 2.13), p-trend 0.02], compared to those of the lowest tertile. However, in the fullyadjusted model, this association became non-significant $[1.41,(0.99,2.01)$ p-trend 0.06]. As regards milk consumption, participants who consumed any amount had a higher risk of falls [fully-adjusted HR $1.53(1.13,2.08)]$ compared to non-consumers. We did not find any statistically significant association between the consumption of yogurt or cheese and the risk of falls by comparing the highest vs. the lowest tertile. Also, when we excluded participants with cardiovascular disease, hip fracture and those who were heavy alcohol drinkers at baseline, we found a null association between the consumption of total dairy and the risk of falls; of note, the positive association between milk consumption and the risk of falls found in the total population disappeared in this subsample (Supplemental table 7).

Finally, when analyzing the relation between total dairy and the risk of falls using nonparametric techniques, some differences between the two cohorts were observed.

Whereas in the Seniors-ENRICA cohort there was no suggestion of a trend, in the UK Biobank, a statistically significant increased risk of falls in those participants who consumed >1.5 servings/day was observed (Supplemental figure 2). 


\section{DISCUSSION}

260 In this study using data from two European cohorts, we generally did not find an

261 association between the different types of dairy products and the risk of falls,

262 independently of population characteristics or among subgroups of participants. There

263 was a suggestion of an increased risk of falls among those who consumed milk in the

264 UK Biobank; however, this result has to be confirmed in future studies.

265 In the scientific literature, we have only identified one study that examined the

266 association between the consumption of dairy products and the risk of falling (14). In

267 this cross-sectional study, no effect of dairy on falls was observed (14). However,

268 authors found a protective association when results were adjusted for non-dairy

269

proteins. Moreover, in a recent systematic review and meta-analysis including 18

270

observational studies, Bian et al. (21) found that individuals with higher consumption of

yogurt and cheese had a lower risk of hip fracture, a serious consequence of falls.

272 Nevertheless, as in our results from the UK Biobank cohort, the authors found a nonlinear positive dose-response association between milk intake and the risk of hip fracture across a range of milk consumption from 0 to $600 \mathrm{~g} /$ day (21). Therefore, our results in the UK Biobank cohort might be driven by those with very high milk intake, although we could not test this hypothesis due to the low number of study participants with such a high consumption.

On the other hand, in the largest study examining the association between milk intake and the risk of mortality and fractures, Michaëlsson et al. (19) found an increased risk of fractures among women who consumed $\geq 3$ glasses/d of milk. Among the possible explanations for this finding was the D-galactose present in milk, but not in fermented dairy products. In animal studies, D-galactose has been associated with chronic inflammation, increased oxidative stress and accelerated aging (39-41), which are also 
factors that might influence falls risk $(42,43)$. Moreover, milk consumption has been positively associated with biomarkers of inflammation and oxidative stress in men and women (19). However, an alternative explanation for this positive association could be the nutritional quality of the background diet. For example, older adults with dentition problems often have diets with poorer nutritional status and inadequate protein intake (44), so for these people dairy could be an important source of protein. In this case, the results found in the UK Biobank cohort might be explained by reverse causation. This explanation is reinforced by the fact that the sensitivity analyses done after eliminating those participants who had a high baseline risk of falls (participants with CVD, previous hip fracture and heavy alcohol drinkers), showed no association. In addition, Ding et al. (45) suggest that the health effect of replacing dairy products depends on the nature of the food for which it is replaced. In our study, we adjusted the analyses for the overall quality of the diet, but we did not have information about changes in the consumption of dairy products among these participants to examine its impact on health.

The divergences in the results found in both cohorts may also be driven for the characteristics of the participants. In the Seniors-ENRICA, mean age was higher and the prevalence of chronic conditions was more elevated than in the UK Biobank study, which may explain the larger number of incident falls. In addition, the UK Biobank studied association by modulating both, the likelihood of falling because of inadequate urban conditions for the older people and the social support available to avoid situations of high risk in this population. 
One of the strengths of this study was the use of two prospective cohorts from countries with different amount and proportion of consumption of dairy products, as well as different socioeconomic characteristics and lifestyle. Additional strengths were the long follow-up period and the large number of potential confounders that were accounted for in the analyses. Specifically, the adjustment for a diet quality score allowed examining the effect of dairy independently from the diet pattern where this food group was consumed, since it has been suggested that dairy products are nutrient-dense foods that may simply reflect a better diet, as in the UK Biobank (46).

This study also has some limitations. Measurement of dairy consumption relied only on the baseline measurements since dietary information in the UK Biobank study has not been updated during follow-up; therefore, possible changes that may have occurred in participants' diets were not taken into account. However, sensitivity analyses performed in the Seniors-ENRICA cohort showed that results using cumulative consumption of dairy products were quite similar than those performed with the baseline measure of diet. Also the relatively low consumption of skim-fat milk and yogurt did not allow for additional analyses for these categories of dairy products; however, current evidence on high-fat vs. low-fat dairy does not conclusively suggest that any of these groups has a beneficial effect on health compared to the other group (10). The measurement of falls was self-reported and some events could have been missed because of the recall bias. Since dietary information was collected when participants already had 60 years old, this null association may not reflect the effect of dairy consumption throughout the life on the development of long-term diseases with an impact on the risk of falls, such as osteoporosis. Finally, as in any observational study, some residual confounding may persist despite relatively exhaustive adjustment. 
333 In conclusion, our results suggest that habitual dairy consumption in the advanced age is

334 not associated with the risk of falling in older adults and therefore, its consumption does

335 not pose a risk for falls in this population. Whether milk consumption might increase

336 the risk of falls, as observed in the UK Biobank cohort, merits further study.

\section{ACKNOWLEDGEMENTS}

338 This research has been conducted with the use of the UK Biobank Resource under

339 application number 29009. UK Biobank is an open access resource. Bona fide

340 researchers can apply to use the UK Biobank data by registering and applying

341 (http://www.ukbiobank.ac.uk/register-apply/).

\section{STATEMENT OF AUTOSHIP}

343 The authors' contributions were as follows: MMF, EAS and ELG: designed the

344 research; MMF: performed the statistical analyses; all authors: contributed to

345 interpretation of the results; MMF, EAS and ELG: drafted the manuscript; ELG: supervised the conduct of research and had primary responsibility for final content; and approved the final manuscript. 
356 The authors declare that they have no competing interests.

\section{FUNDING SOURCES}

358 This work was supported by FIS grants 16/609 and 16/1512 (Instituto de Salud Carlos

359 III, State Secretary of R+D+I, and FEDER/FSE), the ATHLOS project (EU H2020-

360 Project ID: 635316) and the JPI HDHL (SALAMANDER project). 


\section{REFERENCES}

1. Masud T, Morris RO. Epidemiology of falls. Age Ageing 2001;30(4):3-7.

2. López-Torres Hidalgo J, Grupo ANVITAD. Effect of calcium and vitamin D in the reduction of falls in the elderly: a randomized trial versus placebo. Med Clin (Barc) 2014;142(3):95-102.

3. Ringe JD. The effect of Vitamin D on falls and fractures. Scand J Clin Lab Invest Suppl 2012;243:73-8.

4. Zoltick ES, Sahni S, McLean RR, Quach L, Casey VA, Hannan MT. Dietary protein intake and subsequent falls in older men and women: the Framingham Study. J Nutr Health Aging 2011;15(2):147-52.

5. Ballesteros JM, Struijk EA, Rodriguez-Artalejo F, Lopez-Garcia E. Mediterranean diet and risk of falling in community-dwelling older adults. Clin Nutr 2019; doi:10.1016/j.clnu.2019.02.004.

6. Machado-Fragua MD, Struijk EA, Ballesteros JM, Ortolá R, Rodriguez-Artalejo F, Lopez-Garcia E. Habitual coffee consumption and risk of falls in 2 European cohorts of older adults. Am J Clin Nutr 2019;109(5):1431-38.

7. Ortolá R, Garcia-Esquinas E, Galan I, Guallar-Castillon P, Lopez-Garcia E, Banegas JR, Rodriguez-Artalejo F. Patterns of alcohol consumption and risk of falls in older adults: a prospective cohort study. Osteoporos Int 2017;28(11):3143-52

8. Wijesinha-Bettoni R, Burlingame B. Milk and dairy product composition. In: Muehlhoff E, Bennett A, McMahon D, editors. Milk and dairy products in human nutrition. Roma: Food and Agriculture Organization of the United Nations (FAO); 2013. p.41-90. 
9. Lana A, Rodriguez-Artalejo F, Lopez-Garcia E. Dairy consumption and risk of frailty in older adults: a prospective cohort study. J Am Geriatr Soc 2015;63(9):1852-60.

10. Yu E, Hu FB. Dairy products, dairy fatty acids, and the prevention of cardiometabolic disease: a review of recent evidence. Curr Atheroscler Rep 2018;20(5):24.

11. Dehghan M, Mente A, Rangarajan S, Sheridan P, Mohan V, Iqbal R, Gupta R, Lear S, Wentzel-Viljoen E, Avezum A, et al. Association of dairy intake with cardiovascular disease and mortality in 21 countries from five continents (PURE): a prospective cohort study. Lancet 2018;392(10161):2288-97.

12. Gijsbers L, Ding EL, Malik VS, de Goede J, Geleijnse JM, Soedamah-Muthu SS. Consumption of dairy foods and diabetes incidence: a dose-response metaanalysis of observational studies. Am J Clin Nutr 2016;103(4):1111-24.

13. Vissers LET. Sluijs I, van der Schouw YT, Forouhi NG, Imamura F, Burgess S, Barricarte A, Boeing H, Bonet C, Chirlaque MD, Fagherazzi G, Franks PW, Freisling H, Gunter MJ, Quirós JR, Ibsen DB, Kaaks R, Key T, Khaw KT, Kühn T, Mokoroa O, Nilsson PM, Overvad K, Pala V, Palli D, Panico S, Sacerdote C, Spijkerman AMW, Tjonneland A, Tumino R, Rodriguez-Barranco M, Rolandsson O, Riboli E, Sharp SJ, Langenberg C, Wareham NJ. Dairy product intake and risk of type 2 diabetes in EPIC-interact: a mendelian randomization study. Diabetes Care 2019;42(4):568-75.

14. Radavelli-Bagatini S, Zhu K, Lewis JR, Dhaliwal SS, Prince RL. Association of dairy intake with body composition and physical function in older communitydwelling women. J Acad Nutr Diet 2013;113(12):1669-74. 
15. Radavelli-Bagatini S, Zhu K, Lewis JR, Prince RL. Dairy food intake, peripheral bone structure, and muscle mass in elderly ambulatory women. J Bone Miner Res 2014;29(7):1691-700.

16. Mangano KM, Noel SE, Sahni S, Tucker KL. Higher dairy intakes are associated with higher bone mineral density among adults with sufficient vitamin D status: results from the Boston Puerto Rican Osteoporosis Study. J Nutr 2019;149(1):139-48.

17. Hong H, Kim EK, Lee JS. Effects of calcium intake, milk and dairy product intake, and blood vitamin D level on osteoporosis risk in Korean adults: analysis of the 2008 and 2009 Korea National Health and Nutrition Examination Survey. Nutr Res Pract 2013;7(5):409-17.

18. Park SJ, Jung JH, Kim MS, Lee HJ. High dairy products intake reduces osteoporosis risk in Korean postmenopausal women: A 4 year follow-up study. Nutr Res Pract 2018;12(5):436-42.

19. Michaëlsson K, Wolk A, Langenskiöld D, Basu S, Warensjö Lemming E, Melhus H, Byberg L. Milk intake and risk of mortality and fractures in women and men: cohort studies. BMJ 2014;349:g6015.

20. Yoshida D, Ohara T, Hata J, Shibata M, Hirakawa Y, Honda T, Uchida K, Takasugi S, Kitazono T, Kiyohara Y, Ninomiya T. Dairy consumption and risk of functional disability in an elderly Japanese population: the Hisayama Study. Am J Clin Nutr 2019;109(6):1664-71.

21. Bian S, Hu J, Zhang K, Wang Y, Yu M, Ma J. Dairy product consumption and risk of hip fracture: a systematic review and meta-analysis. BMC Public Health 2018;18(1):165. 
22. Feskanich D, Meyer HE, Fung TT, Bischoff-Ferrari HA, Willett WC. Milk and other dairy foods and risk of hip fracture in men and women. Osteoporos Int 2018;29(2):385-96.

23. Rodriguez-Artalejo F, Graciani A, Guallar-Castillón P, León-Muñoz LM, Zuluaga MC, López-García E, Gutiérrez-Fisac JL, Taboada JM, Aguilera MT, Regidor E, et al. Rationale and methods of the study on nutrition and cardiovascular risk in Spain (ENRICA). Rev Esp Cardiol 2011;64:876-82.

24. Sudlow C, Gallacher J, Allen N, Beral V, Burton P, Danesh J, Downey P, Elliott P, Green J, Landray M, et al. UK Biobank: an open access resource for identifying the causes of a wide range of complex diseases of middle and old age. PLoS Med 2015;12 (3):e1001779.

25. Guallar-Castillón P, Sagardui-Villamor J, Balboa-Castillo T, Sala-Vila A, Ariza Astolfi MJ, Sarrión Pelous MD, León-Muñoz LM, Graciani A, Laclaustra M, Benito C, et al. Validity and reproducibility of a Spanish dietary history. PloS One 2014;9:e86074.

26. Willett WC. Nutritional Epidemiology. Thrid Edition. New York, NY: Oxford University Press, 2012.

27. Schröder H, Fitó M, Estruch R, Martinez-Gonzalez MA, Corella D, SalasSalvadó J, Lamuela-Raventós R, Ros E, Salaverria I, Fiol M, Lapetra J, Vinyoles E, Gomez-Gracia E, Lahoz C, Serra-Majem L, Pintó X, Ruiz-Gutierrez V, Covas MI. A short screener is valid for assessing Mediterranean diet adherence among older Spanish men and women. J Nutr 2011;141(6):1140-5.

28. Galante J, Adamska L, Young A, Young H, Littlejohns TJ, Gallacher J, Allen N. The acceptability of repeat Internet-based hybrid diet assessment of previous 24- 
h dietary intake: administration of the Oxford WebQ in UK Biobank. Br J Nutr 2016;115(4):681-6.

29. Holland B, Welch A, Unwin I, Buss DH, Paul AA, Southgate DAT (1991). McCance and Widdowson's. The Composition of Foods. 5th edition. Cambridge: Royal Society of Chemistry.

30. Martínez-Gómez D, Guallar-Castillón P, León-Muñoz LM, López-García E, Rodríguez-Artalejo F. Combined impact of traditional and non-traditional health behaviors on mortality: a national prospective cohort study in Spanish older adults._BMC Med 2013;11:47.

31. Wade KH, Carslake D, Sattar N, Davey Smith G, Timpson NJ. BMI and Mortality in UK Biobank: Revised Estimates Using Mendelian Randomization. Obesity (Silver Spring) 2018;26(11):1796-1806.

32. Ortolá R, García-Esquinas E, Galán I, Guallar-Castillón P, López-García E, Banegas JR, Rodríguez-Artalejo F. Patterns of alcohol consumption and risk of falls in older adults: a prospective cohort study. Osteoporos Int 2017:28(11):3143-52

33. Pols MA, Peeters PH, Ocke MC, Slimani N, Bueno-de-Mesquita HB, Collette HJ. Estimation of reproducibility and relative validity of the questions included in the EPIC Physical Activity Questionnaire. Int J Epidemiol 1997;26:S181-9.

34. Morley JE, Malmstrom TK, Miller DK. A simple frailty questionnaire (FRAIL) predicts outcomes in middle aged African Americans. J Nutr Health Aging 2012;16:601-608.

35. Janssen I, Heymsfield SB, Baumgartner RN, Ross R. Estimation of skeletal muscle mass by bioelectrical impedance analysis. J Appl Physiol (1985). 2000;89(2):465-71. 
36. Guralnik JM, Ferrucci L, Pieper CF, Leveille SG, Markides KS, Ostir GV, et al. Lower extremity function and subsequent disability: consistency across studies, predictive models, and value of gait speed alone compared with the short physical performance battery. J Gerontol A Biol Sci Med Sci. 2000;55:M221231.

37. Craig CL, Marshall AL, Sjöström M, Bauman AE, Booth ML, Ainsworth BE, Pratt M, Ekelund U, Yngve A, Sallis JF, et al. International physical activity questionnaire: 12-country reliability and validity. Med Sci Sports Exerc 2003;35(8):1381-95.

38. Kojima G. Frailty as a predictor of future falls among community-dwelling older people: a systematic review and meta-analysis. J Am Med Dir Assoc 2015;16(12):1027-33.

39. Song X, Bao M, Li D, Li YM. Advanced glycation in D-galactose induced mouse aging model. Mech Ageing Dev 1999;108:239-51.

40. Cui X, Zuo P, Zhang Q, Li X, Hu Y, Long J. Chronic systemic D-galactose exposure induces memory loss, neurodegeneration, and oxidative damage in mice: protective effects of R-alpha-lipoic acid. J Neurosci Res 2006;83:1584-90.

41. Hao L, Huang H, Gao J, Marshall C, Chen Y, Xiao M. the influence of gender, age and treatment time on brain oxidative stress and memory impairment induced by d-galactose in mice. Neurosci Lett 2014;571C:45-9.

42. Britton GB, O’Bryant SE, Johnson LA, Hall JR, Villareal AE, Oviedo DC, Lao ARP, Carreira MB, For The Panama Aging Research Initiative SG.

Inflammatory biomarkers, depressive symptoms and falls among the elderly in Panama. Curr Aging Sci 2019;11(4):236-41. 
43. Verghese J, Ayers E. Biology of falls: preliminary cohort study suggesting a possible role for oxidative stress. J Am Geriatr Soc 2017;65(6):1306-9.

44. Saarela RK, Lindroos E, Soini H, Hiltunen K, Muurinen S, Suominen MH, Pitkälä KH. Dentition, nutritional status and adequacy of dietary intake among older residents in assisted living facilities. Gerontology 2016;33(2):225-32.

45. Ding M, Li J, Qi L, Ellervik C, Zhang X, Manson JE, Stampfer M, Chavarro JE, Rexrode KM, Kraft P, et al. Associations of dairy intake with risk of mortality in women and men: three prospective cohort studies. BMJ 2019;367:I6204.

46. van Staveren WA, Steijns JM, de Groot LC. Dairy products as essential contributors of (micro-) nutrients in reference food patterns: an outline for elderly people. J Am Coll Nutr 2008;27(6):747S-54S. 
Table 1. Baseline characteristics of participants across tertiles of dairy consumption in the Seniors-ENRICA and in the UK Biobank study.

\begin{tabular}{|c|c|c|c|c|c|c|}
\hline & \multicolumn{3}{|c|}{ Seniors-ENRICA study $(\mathrm{N}=2,981)$} & \multicolumn{3}{|c|}{ UK Biobank study $(\mathrm{N}=8,927)$} \\
\hline & $\begin{array}{l}\text { Tertile } 1 \\
(0-1.62)\end{array}$ & $\begin{array}{c}\text { Tertile } 2 \\
(1.63-2.62)\end{array}$ & $\begin{array}{c}\text { Tertile } 3 \\
(2.63-12.0)\end{array}$ & $\begin{array}{l}\text { Tertile } 1 \\
(0-0.60)\end{array}$ & $\begin{array}{c}\text { Tertile } 2 \\
(0.61-1.12)\end{array}$ & $\begin{array}{c}\text { Tertile } 3 \\
(1.13-6.50)\end{array}$ \\
\hline Participants, $n$ & 994 & 994 & 993 & 3224 & 2728 & 2975 \\
\hline Age, $y$ & $68.7(6.5)$ & $69.1(6.5)$ & $69.4(6.7)$ & $63.7(2.7)$ & $63.7(2.7)$ & $63.7(2.5)$ \\
\hline Men, \% & 49.7 & 45.5 & $43.7^{*}$ & 58.6 & 50.0 & $49.9^{* *}$ \\
\hline \multicolumn{7}{|l|}{ Educational level, \% } \\
\hline$\leq$ Primary & 55.9 & 58.5 & 57.2 & 11.7 & 9.5 & 7.1 \\
\hline Secondary & 23.9 & 22.6 & 24.4 & 30.6 & 28.8 & 28.3 \\
\hline University & 20.2 & 18.9 & 18.4 & 57.7 & 61.7 & $64.6^{5}$ \\
\hline Current smoker, \% & 14.7 & 13.1 & $8.2^{5}$ & 5.9 & 5.2 & $3.8^{5}$ \\
\hline Heavy drinker ${ }^{\mathrm{a}}, \%$ & 10.5 & 7.6 & $5.9^{4}$ & 19.1 & 16.6 & $15.7^{4}$ \\
\hline BMI, $\mathrm{kg} / \mathrm{m}^{2}$ & $28.4(4.4)$ & $28.4(4.3)$ & $28.9(4.6)^{*}$ & $26.7(4.2)$ & $26.4(3.9)$ & $26.7(4.4)$ \\
\hline Physical activity, METs-h/wk & $22.0(15.8)$ & $21.7(14.8)$ & $21.1(15.0)$ & $43.3(50.2)$ & $42.6(50.7)$ & $43.7(45.5)$ \\
\hline Sleep duration, h/d & $6.9(1.4)$ & $6.9(1.4)$ & $6.8(1.4)$ & $7.3(1.0)$ & $7.3(0.9)$ & $7.3(0.9)$ \\
\hline Energy intake, $\mathrm{kcal} / \mathrm{d}$ & $1900(562)$ & 2049 (673) & $2109(749)^{* *}$ & $2043(479)$ & $2088(464)$ & $2206(499)^{* *}$ \\
\hline Intake of calcium, mg/dl & $615(167)$ & $843(158)$ & $1182(342)^{* *}$ & $848(261)$ & $970(245)$ & $1141(292)^{* *}$ \\
\hline Intake of protein, $\mathrm{g} / \mathrm{d}$ & $84.1(25.0)$ & $90.2(24.7)$ & $98.6(28.0)^{* * *}$ & $78.4(18.2)$ & $81.4(17.8)$ & $87.9(19.6)^{* *}$ \\
\hline Intake of saturated fat, $g / d$ & $20.6(8.9)$ & $24.1(9.9)$ & $28.4(12.9)^{* *}$ & $28.6(10.1)$ & $29.4(10.2)$ & $31.8(10.8)^{*}$ \\
\hline MEDAS score & $7.0(1.7)$ & $6.7(1.9)$ & $6.8(1.7)^{*}$ & $4.3(1.5)$ & $4.4(1.6)$ & $4.6(1.6)^{* * *}$ \\
\hline \multicolumn{7}{|l|}{ Diagnosed diseases, $\%$} \\
\hline Hypertension & 63.2 & 64.6 & 65.3 & 35.3 & 33.8 & 33.2 \\
\hline Diabetes & 17.0 & 16.4 & 15.7 & 5.2 & 4.1 & 4.4 \\
\hline Osteomuscular disease & 48.2 & 49.8 & 49.9 & 22.1 & 21.7 & 23.0 \\
\hline Use of sleeping pills, \% & 13.6 & 14.1 & 16.3 & 0.5 & 0.4 & 0.5 \\
\hline Muscle mass in \%, year 2013 & $49.4(8.9)$ & $49.0(9.2)$ & $48.8(9.0)$ & - & - & - \\
\hline Exhaustion, \%, year 2013 & 4.5 & 5.9 & 5.3 & - & - & - \\
\hline Slow walking speed, \%, year 2013 & 9.8 & 10.1 & 10.2 & - & - & - \\
\hline
\end{tabular}

Note: MET: Metabolic Equivalent. BMI: Body Mass Index

For continuous variables, the mean (standard deviation) is reported.

${ }^{a}$ Heavy drinker: $\geq 40 \mathrm{~g} / \mathrm{d}$ of alcohol in men and $\geq 24 \mathrm{~g} / \mathrm{d}$ in women.

${ }^{\mathrm{b}}$ Serving sizes in Seniors-ENRICA study ( 1 serving): $200 \mathrm{ml}$ of milk, $125 \mathrm{~g}$ of yogurt, and $40 \mathrm{~g}$ of cheese.

${ }^{\mathrm{c}}$ Serving sizes in UK Biobank study (1 serving): $250 \mathrm{ml}$ of milk, $125 \mathrm{~g}$ of yogurt, and $40 \mathrm{~g}$ of cheese.

" $\mathrm{p}<0.05 ;{ }^{* *} \mathrm{p}<0.001$. 
Table 2. Dairy consumption in the Seniors-ENRICA and UK Biobank study.

\begin{tabular}{lcc}
\hline & Seniors-ENRICA study $(\mathrm{N}=2,981)$ & UK Biobank study $(\mathrm{N}=8,927)$ \\
\cline { 2 - 3 } & Mean $(\mathrm{SD})$ servings/d & Mean $(\mathrm{SD})$ servings/d \\
\hline Total dairy products & $2.28(1.36)$ & $0.92(0.64)$ \\
Milk & $1.03(0.80)$ & $0.18(0.30)$ \\
Whole milk & $0.23(0.53)$ & $0.01(0.09)$ \\
Part-skim milk & $0.41(0.65)$ & $0.12(0.26)$ \\
Skim milk & $0.39(0.72)$ & $0.05(0.16)$ \\
Yogurt & $0.59(0.66)$ & $0.41(0.45)$ \\
Full-fat yogurt & $0.25(0.49)$ & $0.08(0.23)$ \\
Low-fat yogurt & $0.34(0.57)$ & $0.33(0.43)$ \\
Cheese & $0.66(0.87)$ & $0.33(0.31)$ \\
\hline
\end{tabular}


Table 3. Hazard ratios ( $95 \%$ confidence interval) for the association between dairy consumption and the risk of $\geq 1$ falls in the Seniors-ENRICA study $(\mathrm{N}=2,981)$.

\begin{tabular}{|c|c|c|c|c|c|}
\hline & \multicolumn{4}{|c|}{ Dairy consumption, servings/d } & \multirow{2}{*}{$\begin{array}{c}\text { Continuous per } 1 \\
\text { serving/d } \\
\text { increment }\end{array}$} \\
\hline & Tertile 1 & Tertile 2 & Tertile 3 & $\begin{array}{l}\mathrm{P}- \\
\text { trend }\end{array}$ & \\
\hline \multicolumn{6}{|l|}{ Total dairy } \\
\hline Tertile range & $0-1.62$ & $1.63-2.62$ & $2.63-12.01$ & & \\
\hline Person-years & 5014 & 4961 & 4952 & & \\
\hline Cases, $\mathrm{n}$ & 261 & 257 & 283 & & \\
\hline Age- and sex-adjusted model & 1.00 & $0.99(0.83-1.17)$ & $1.09(0.92-1.29)$ & 0.32 & $1.04(0.96-1.14)$ \\
\hline Multivariable $^{1}$ & 1.00 & $0.99(0.83-1.17)$ & $1.03(0.86-1.22)$ & 0.75 & $1.01(0.93-1.11)$ \\
\hline Multivariable $^{2}$ & 1.00 & $0.99(0.83-1.18)$ & $1.03(0.87-1.23)$ & 0.72 & $1.02(0.93-1.11)$ \\
\hline \multicolumn{6}{|l|}{ Milk } \\
\hline Tertile range & $0-0.68$ & $0.69-1.21$ & $1.22-7.84$ & & \\
\hline Person-years & 4932 & 5115 & 4880 & & \\
\hline Cases, $\mathrm{n}$ & 275 & 279 & 247 & & \\
\hline Age- and sex-adjusted model & 1.00 & $0.88(0.75-1.04)$ & $0.89(0.75-1.06)$ & 0.19 & $0.94(0.86-1.03)$ \\
\hline Multivariable $^{1}$ & 1.00 & $0.86(0.72-1.01)$ & $0.84(0.71-1.01)$ & 0.06 & $0.92(0.84-1.00)$ \\
\hline Multivariable $^{2}$ & 1.00 & $0.85(0.72-1.01)$ & $0.85(0.71-1.02)$ & 0.08 & $0.92(0.84-1.01)$ \\
\hline Multivariable ${ }^{3}$ & 1.00 & $0.86(0.73-1.02)$ & $0.86(0.72-1.03)$ & 0.10 & $0.93(0.85-1.01)$ \\
\hline \multicolumn{6}{|l|}{ Yogurt } \\
\hline Tertile range & 0 & $0.01-0.92$ & $0.93-7.00$ & & \\
\hline Person-years & 5390 & 4916 & 4621 & & \\
\hline Cases, $\mathrm{n}$ & 274 & 256 & 271 & & \\
\hline Age- and sex-adjusted model & 1.00 & $0.96(0.81-1.14)$ & $1.11(0.94-1.32)$ & 0.22 & $1.06(0.97-1.15)$ \\
\hline Multivariable $^{1}$ & 1.00 & $0.97(0.82-1.16)$ & $1.09(0.92-1.30)$ & 0.32 & $1.05(0.96-1.14)$ \\
\hline Multivariable $^{2}$ & 1.00 & $0.96(0.81-1.14)$ & $1.09(0.92-1.30)$ & 0.34 & $1.04(0.96-1.14)$ \\
\hline Multivariable ${ }^{3}$ & 1.00 & $0.97(0.81-1.16)$ & $1.10(0.92-1.31)$ & 0.29 & $1.05(0.96-1.15)$ \\
\hline \multicolumn{6}{|l|}{ Cheese } \\
\hline Tertile range & $0-0.15$ & $0.16-0.68$ & $0.69-9.43$ & & \\
\hline Person-years & 5002 & 4904 & 5021 & & \\
\hline Cases, $\mathrm{n}$ & 277 & 255 & 269 & & \\
\hline Age- and sex-adjusted model & 1.00 & $0.93(0.78-1.10)$ & $0.97(0.82-1.15)$ & 0.74 & $0.99(0.91-1.07)$ \\
\hline Multivariable $^{1}$ & 1.00 & $0.93(0.78-1.11)$ & $0.93(0.78-1.11)$ & 0.41 & $0.96(0.88-1.05)$ \\
\hline Multivariable $^{2}$ & 1.00 & $0.92(0.78-1.10)$ & $0.92(0.77-1.10)$ & 0.36 & $0.96(0.88-1.05)$ \\
\hline Multivariable $^{3}$ & 1.00 & $0.93(0.78-1.11)$ & $0.93(0.78-1.11)$ & 0.42 & $0.96(0.88-1.05)$ \\
\hline
\end{tabular}

${ }^{1}$ Cox model adjusted for age, sex, educational level (sprimary, secondary, university), smoking status (never smoker, former smoker, current smoker), alcohol consumption (abstainer, moderate, heavy drinker), BMI (tertiles of kg/m²), physical activity (tertiles of MET-h/wk), sleep duration (tertiles of h/d), energy intake (tertiles of kcal/d), MEDAS score (tertiles), hypertension, diabetes, and use of sleeping pills.

${ }^{2}$ Cox model additionally adjusted for osteomuscular disease.

${ }^{3}$ Cox model additionally adjusted for the other types of dairy products. 
Table 4. Hazard ratios ( $95 \%$ confidence interval) for the association between dairy consumption and the risk of $\geq 1$ falls in the UK Biobank study $(\mathrm{N}=8,927)$.

\begin{tabular}{|c|c|c|c|c|c|}
\hline & \multicolumn{4}{|c|}{ Dairy consumption, servings/d } & \multirow{2}{*}{$\begin{array}{c}\text { Continuous per } 1 \\
\text { serving/d } \\
\text { increment }\end{array}$} \\
\hline & Tertile 1 & Tertile 2 & Tertile 3 & $\begin{array}{c}\mathrm{P}- \\
\text { trend }\end{array}$ & \\
\hline \multicolumn{6}{|l|}{ Total dairy } \\
\hline Tertile range & $0-0.60$ & $0.61-1.12$ & $1.13-6.50$ & & \\
\hline Person-years & 12168 & 9920 & 10184 & & \\
\hline Cases, $\mathrm{n}$ & 64 & 68 & 69 & & \\
\hline Age- and sex-adjusted model & 1.00 & $1.32(0.93-1.86)$ & $1.51(1.07-2.13)$ & 0.02 & $1.23(1.04-1.45)$ \\
\hline Multivariable $^{1}$ & 1.00 & $1.31(0.93-1.86)$ & $1.44(1.01-2.06)$ & 0.04 & $1.20(1.01-1.43)$ \\
\hline Multivariable $^{2}$ & 1.00 & $1.31(0.93-1.85)$ & $1.41(0.99-2.01)$ & 0.06 & $1.19(1.00-1.41)$ \\
\hline \multicolumn{6}{|l|}{ Milk } \\
\hline Consumption (yes vs no) & No & & Yes & & \\
\hline Person-years & 22581 & & 9692 & & \\
\hline Cases, $\mathrm{n}$ & 135 & & 66 & & \\
\hline Age- and sex-adjusted model & 1.00 & - & $1.57(1.16-2.11)$ & & \\
\hline Multivariable $^{1}$ & 1.00 & - & $1.57(1.16-2.13)$ & & \\
\hline Multivariable $^{2}$ & 1.00 & - & $1.54(1.14-2.09)$ & & \\
\hline Multivariable $^{3}$ & 1.00 & - & $1.53(1.13-2.08)$ & & \\
\hline \multicolumn{6}{|l|}{ Yogurt } \\
\hline Tertile range & 0 & $0.25-0.50$ & $0.60-3.00$ & & \\
\hline Person-years & 13942 & 8434 & 9896 & & \\
\hline Cases, $\mathrm{n}$ & 69 & 68 & 64 & & \\
\hline Age- and sex-adjusted model & 1.00 & $1.47(1.04-2.06)$ & $1.22(0.87-1.73)$ & 0.23 & $1.11(0.94-1.31)$ \\
\hline Multivariable $^{1}$ & 1.00 & $1.49(1.05-2.10)$ & $1.22(0.86-1.75)$ & 0.24 & $1.11(0.93-1.31)$ \\
\hline Multivariable $^{2}$ & 1.00 & $1.48(1.05-2.09)$ & $1.21(0.85-1.73)$ & 0.27 & $1.10(0.93-1.31)$ \\
\hline Multivariable ${ }^{3}$ & 1.00 & $1.45(1.02-2.06)$ & $1.21(0.85-1.73)$ & 0.28 & $1.10(0.93-1.31)$ \\
\hline \multicolumn{6}{|l|}{ Cheese } \\
\hline Tertile range & $0-0.20$ & $0.30-0.40$ & $0.50-2.60$ & & \\
\hline Person-years & 16167 & 7552 & 8553 & & \\
\hline Cases, $\mathrm{n}$ & 100 & 44 & 57 & & \\
\hline Age- and sex-adjusted model & 1.00 & $1.00(0.70-1.42)$ & $1.18(0.85-1.64)$ & 0.35 & $1.08(0.92-1.27)$ \\
\hline Multivariable $^{1}$ & 1.00 & $0.95(0.66-1.36)$ & $1.05(0.75-1.47)$ & 0.82 & $1.02(0.86-1.21)$ \\
\hline Multivariable $^{2}$ & 1.00 & $0.98(0.68-1.40)$ & $1.09(0.78-1.53)$ & 0.65 & $1.04(0.88-1.23)$ \\
\hline Multivariable $^{3}$ & 1.00 & $1.01(0.70-1.45)$ & $1.07(0.76-1.50)$ & 0.70 & $1.03(0.87-1.22)$ \\
\hline
\end{tabular}

${ }^{1}$ Cox model adjusted for age, sex, educational level (sprimary, secondary, university), smoking status (never smoker, former smoker, current smoker), alcohol consumption (abstainer, moderate, heavy drinker), BMI (tertiles of kg/m²), physical activity (tertiles of MET-h/wk), sleep duration (tertiles of h/d), energy intake (tertiles of kcal/d), MEDAS score (tertiles), hypertension, diabetes, and use of sleeping pills.

${ }^{2}$ Cox model additionally adjusted for osteomuscular disease.

${ }^{3}$ Cox model additionally adjusted for the other types of dairy products. 